\title{
La roxitromicina disminuyó los eventos coronarios en pacientes con angina inestable
}

Gurfinkel E, Bozovich E, Daroca A. Randomised trial of roxithromycin in non-Q-wave coronary syndromes: Roxis pilot study. Lancet 1997; 350:404-407.

\section{Objetivo}

Evaluar si la roxitromicina previene o reduce el riesgo de sufrir eventos coronarios en pacientes con angina inestable.

\section{Diseño}

Ensayo clínico Piloto Multicéntrico, doble ciego, randomizado y controlado.

\section{Lugar: \\ 8 Unidades de cuidados Intensivos Coronarios en Ar- gentina \\ Pacientes \\ 202 pacientes admitidos a unidades coronarias por epi- sodios de angina inestable o IAM no Q de menos de 48 hs de evolución con evidencia de isquemia electrocardiografica o enzimática o antecedentes de enfermedad coronaria. Fueron excluídos pacientes con IAM tipo Q en evolución, contrain- dicación para uso de macrólidos, falla hepática o renal o blo- queo completo de rama izquierda.}

\section{Intervencion}

Roxitromicina $150 \mathrm{mg}$ cada 12 hs por 30 dias ( $\mathrm{n}=100)$ o placebo $(n=102)$ agregado al tratamiento habitual de la angina inestable.

\section{Medición de Resultados principales}

Se consideró un evento principal combinado definido por la ocurrencia de alguno de los siguientes a los 30 días: angina severa recurrente, IAM o muerte de causa isquémica.

\section{Resultados Principales}

En el análisis por intención de tratar, 2 pacientes en el grupo roxitromicina y 9 en el grupo placebo sufrieron al menos uno de los tres eventos considerados $(\mathrm{p}=0.06)$. Para el análisis de eficacia se consideraron sólo aquellos que cumplimentaron al menos 3 días de tratamiento (93 pacientes en cada grupo), la diferencia en este subgrupo resultó estadísticamente significativa $(p=0,036)$ demostrando también una reducción no significativa en el riesgo de angina recurrente $(5,4 \%$ vs $1,1 \%)$, IAM $(2,2 \%$ vs $0 \%)$ y muerte $(2,2 \%$ vs $0 \%)$.

\section{Conclusiones}

El agregado de roxitromicina durante 30 días al tratamiento habitual de los sindromes isquémicos coronarios sin onda $Q$, disminuyó la incidencia precoz de IAM, angina recurrente o muerte de causa isquémica.

Apoyo económico: Laboratorios Hoechst Marion Russell

\section{Comentario}

Varios estudios caso control han reportado una asociación significativa entre la presencia de títulos positivos de anticuerpos IgG anti clamidia pneumoniae en pacientes con IAM u otros eventos coronarios. Este es un interesante estudio piloto Argentino que explora la eficacia del uso de roxitromicina, un eficaz agente anticlamidia, en el tratamiento de la enfermedad coronaria aguda. Los autores eligen pacientes con angina inestable o IAM no Q como blanco de su estudio por ser quienes más probablemente desarrollen alguno de los eventos considerados como resultado principal y en los que los fenómenos inflamatorios endoteliales juegan presumiblemente un rol importante. No queda claro, sin embargo, la elección de un n de 200 pacientes cuando los cálculos para un estudio randomizado de las características expuestas por los mismos autores (incidencia del evento de $14 \%$ y una reducción estimada con roxi- tromicina del 20\%) exige un numero 20 veces mayor. La inesperada gran diferencia en la ocurrencia del evento combinado en los grupos ( $9 \%$ en el grupo placebo y 1 o $2 \%$ en el experimental) permitió ver diferencias significativas con solo 200 pacientes. Sin embargo, el hecho de basarse en 1 o 2 eventos puede hacer inestables las estimaciones estadísticas ya que si el grupo roxitromicina hubiera tenido 1 sólo evento más, la diferencia no habría sido estadísticamente significativa. La necesidad de cirugía de revascularización coronaria durante el seguimiento a partir del $3 \mathrm{er}$ día ( 16 en el grupo tratamiento y 12 en el placebo), no se evaluó en los resultados. Si se hubiera considerado como un evento más, la diferencia entre los grupos tampoco hubiera sido significativa ( $R R$ calculado $0,81$, IC $95 \% 0,46-1,43)$. Si bien todavía no esta definido si la Chlamidia Pneumoniae juega algún rol en la patogénesis de la injuria endote- lial, esta asociación epidemiológica y los resultados preliminares de este estudio son muy sugestivos. Es realmente estimulante ver un estudio realizado por un grupo Argentino que abre una nueva línea de investigación en la terapéutica de la enfermedad coronaria.

Dr. Juan P. Roubicek Clínica Médica Hospital Privado de la Comunidad. Mar del Plata

\section{Dr. Fernando Rubinstein} Unidad de Medicina Familiar y Preventiva Hospital Italiano de Buenos Aires

\section{Referencias}

1.Danesh J, Collins R, Peto R. Chronic infections and coronary heart disease: is there a link? Lancet 1997;350:430-6

2.Lip GY, Beevers DG. Can we treat coronary artery disease with antibiotics? Lancet 1997:350:378-9 